\title{
Measurements of keV-neutron capture cross sections and capture gamma-ray spectra for $\mathrm{Sn}$ and $\mathrm{Gd}$ isotopes
}

\author{
J. Nishiyama ${ }^{1, a}$, T.I. Ro ${ }^{2}$, M. Igashira ${ }^{1}$, W.C. Chung ${ }^{2}$, G. Kim ${ }^{3}$, T. Ohsaki ${ }^{1, b}$, S. Lee $^{4}$, and T. Katabuchi ${ }^{1}$ \\ 1 Research Laboratory for Nuclear Reactors, Tokyo Institute of Technology, Tokyo 152-8550, Japan \\ 2 Department of Physics, Dong-A University, Busan 604-714, Korea \\ 3 Department of Physics, Kyungpook National University, Daegu 702-701, Korea \\ 4 Department of Radiological Science, Nambu University, Gwangju 506-824, Korea
}

\begin{abstract}
The neutron capture cross sections and capture gamma-ray spectra of Sn $\left({ }^{116-119} \mathrm{Sn}\right)$ and $\mathrm{Gd}\left({ }^{155-158} \mathrm{Gd}\right)$ isotopes have been measured in the incident neutron energy region from 10 to $100 \mathrm{keV}$ and at $550 \mathrm{keV}$. Pulsed keV neutrons were produced by the ${ }^{7} \mathrm{Li}(\mathrm{p}, \mathrm{n})^{7} \mathrm{Be}$ reaction. Capture gamma rays were detected with a large anti-Compton $\mathrm{NaI}(\mathrm{Tl})$ spectrometer by means of a time-of-flight method. A pulse-height weighting technique was applied to the observed capture gamma-ray pulse-height spectra to obtain capture yields. Using the standard capture cross sections of ${ }^{197} \mathrm{Au}$, the capture cross sections of the $\mathrm{Sn}$ and $\mathrm{Gd}$ isotopes were derived. The capture gamma-ray spectra were obtained by unfolding the observed capture gamma-ray pulse-height spectra. The present cross section results were compared with other experimental data and evaluated values.
\end{abstract}

\section{Introduction}

Neutron capture cross sections and capture gamma-ray spectra are important quantities in the $R \& D$ of innovative nuclear reactors.

Those of long-lived fission products (LLFPs) are significant for the R\&D of nuclear transmutation systems, because the performance of system using neutron capture reaction depends directly on these quantities. The nuclide ${ }^{126} \mathrm{Sn}$ is one of LLFPs. However, the present status of experimental data for the neutron cross sections of ${ }^{126} \mathrm{Sn}$ is very poor both in quality and in quantity: only one data was reported at thermal energy [1], because the preparation of high-purity sample is difficult and, moreover, the gamma-ray radiation from a sample causes a serious background in the cross section measurement. On the other hand, keV-neutron capture cross sections and capture gamma-ray spectra of stable $\mathrm{Sn}$ isotopes contain important information useful for the theoretical calculation of capture cross sections of ${ }^{126} \mathrm{Sn}$.

The neutron capture cross sections of Gd isotopes are important in the design of thermal and fast reactors, as well as in nuclear reaction and astrophysics studies. Since a major portion of the natural $\mathrm{Gd}$ capture cross sections is due to ${ }^{155} \mathrm{Gd}$ and ${ }^{157} \mathrm{Gd}$, an accurate knowledge of the cross sections for these isotopes is of importance for calculating the reactor characteristics when $\mathrm{Gd}$ is used as a burnable poison in light water reactors. In addition, the data are important for examining the availability of Gd as a control material for fast reactors.

Therefore, we are performing the systematic measurement of keV-neutron capture cross sections and capture gamma-ray spectra for stable $\mathrm{Sn}$ and $\mathrm{Gd}$ isotopes. In the present paper, we report on the capture cross sections and capture gamma-ray spectra of ${ }^{116,117,118,119} \mathrm{Sn}$ and ${ }^{155,156,157,158} \mathrm{Gd}$.

${ }^{a}$ Presenting author, e-mail: jun-nishiyama@nr.titech.ac.jp

${ }^{b}$ Present address: Intellectual Property Bank Corp., Tokyo, Japan

\section{Experimental procedure}

The experimental procedure has been described in detail elsewhere [2], so it is summarized here.

The capture cross sections and capture gamma-ray spectra of ${ }^{116,117,118,119} \mathrm{Sn}$ and ${ }^{155,156,157,158} \mathrm{Gd}$ were measured in the incident neutron energy region from 10 to $100 \mathrm{keV}$ and at $550 \mathrm{keV}$, using the 3-MV Pelletron accelerator of the Research Laboratory for Nuclear Reactors at the Tokyo Institute of Technology. An experimental arrangement is shown in figure 1. Pulsed neutrons were produced by the ${ }^{7} \mathrm{Li}(\mathrm{p}, \mathrm{n}){ }^{7} \mathrm{Be}$ reaction with a pulsed proton beam $(1.5 \mathrm{~ns}$ width, $4 \mathrm{MHz}$ repetition rate) from the Pelletron accelerator. The incident neutron spectrum on a capture sample was measured by means of a time-of-flight (TOF) method with a ${ }^{6} \mathrm{Li}$-glass scintillation detector. A $5.0-\mathrm{mm}$ diameter by $5.0-\mathrm{mm}{ }^{6} \mathrm{Li}$-glass detector located $30 \mathrm{~cm}$ from the neutron source was used for the measurements in the $10-100 \mathrm{keV}$ region, and a $102.0-\mathrm{mm}$ diameter by $6.4-\mathrm{mm}{ }^{6} \mathrm{Li}$-glass detector located $460 \mathrm{~cm}$ was used for those at $550 \mathrm{keV}$.

Each of ${ }^{116,117,118,119} \mathrm{Sn}$ and ${ }^{155,156,157,158} \mathrm{Gd}$ samples was isotopically enriched metal, and the net weight of each sample was $0.2-1.0 \mathrm{~g}$. The characteristics of the samples are shown in table 1.

A gold (Au) sample was used as a standard. Each capture sample was located at an angle of 0 degree with respect to the proton beam direction. The distance between the neutron source and the sample was $12 \mathrm{~cm}$ for the measurements in the $10-100 \mathrm{keV}$ region and $20 \mathrm{~cm}$ for those at $550 \mathrm{keV}$.

The capture gamma rays emitted from the sample were measured with a large anti-Compton $\mathrm{NaI}(\mathrm{Tl})$ spectrometer by means of a TOF method. The main $\mathrm{NaI}(\mathrm{Tl})$ detector of the spectrometer had a diameter of $15.2 \mathrm{~cm}$ and a length of $20.3 \mathrm{~cm}$ or $30.5 \mathrm{~cm}$. The larger $\mathrm{NaI}(\mathrm{Tl})$ detector had $30 \%$ higher detection efficiency around $5 \mathrm{MeV}$ compared to the smaller detector [3], and was used for the measurements of ${ }^{116,117,118} \mathrm{Sn}$. One of the main detectors was centered in a 


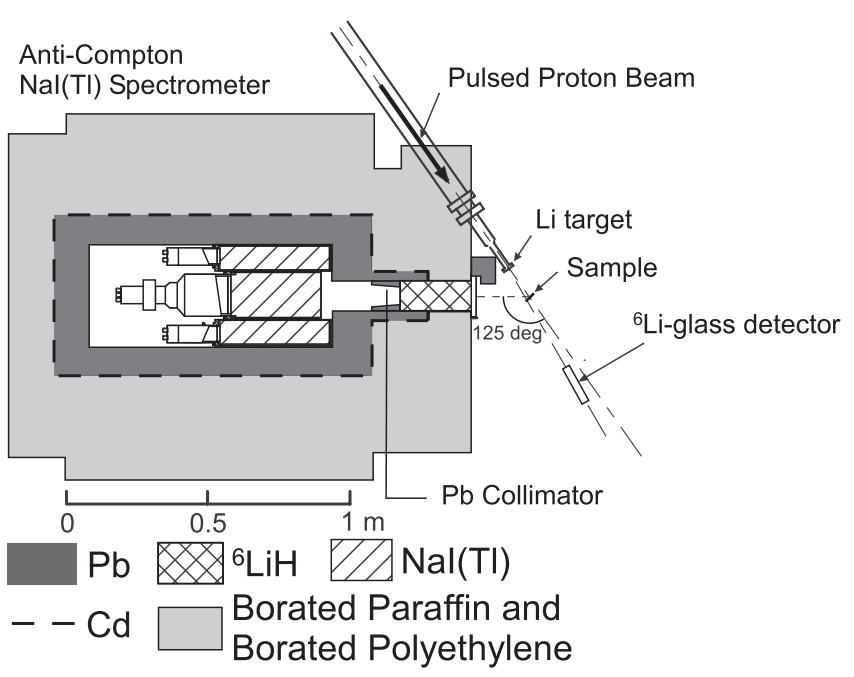

Fig. 1. Experimental arrangement in the $10-100 \mathrm{keV}$ measurement.

Table 1. Characteristics of Sn and Gd samples.

\begin{tabular}{ccccc}
\hline Sample & ${ }^{116} \mathrm{Sn}$ & ${ }^{117} \mathrm{Sn}$ & ${ }^{118} \mathrm{Sn}$ & ${ }^{119} \mathrm{Sn}$ \\
\hline Weight [g] & 0.988 & 1.000 & 0.999 & 0.996 \\
Thickness [mm] & 1.3 & 0.55 & 0.53 & 0.60 \\
Isotopic composition [\%] & & & & \\
${ }^{112} \mathrm{Sn}$ & $<0.01$ & $<0.1$ & 0.07 & $<0.03$ \\
${ }^{114} \mathrm{Sn}$ & 0.2 & $<0.01$ & 0.02 & 0.02 \\
${ }^{115} \mathrm{Sn}$ & 0.48 & $<0.11$ & 0.02 & 0.05 \\
${ }^{116} \mathrm{Sn}$ & 96.1 & 4.4 & 0.48 & 0.11 \\
${ }^{117} \mathrm{Sn}$ & 2.1 & 95.1 & 0.62 & 0.06 \\
${ }^{118} \mathrm{Sn}$ & 0.3 & 0.2 & 96.2 & 0.95 \\
${ }^{119} \mathrm{Sn}$ & 0.2 & $<0.05$ & 0.79 & 93.8 \\
${ }^{120} \mathrm{Sn}$ & 0.5 & $<0.01$ & 0.97 & 4.89 \\
${ }^{122} \mathrm{Sn}$ & 0.1 & $<0.01$ & 0.71 & 0.05 \\
${ }^{124} \mathrm{Sn}$ & $<0.01$ & $<0.01$ & 0.12 & 0.04 \\
\hline${ }^{\mathrm{Sample}}$ & ${ }^{155} \mathrm{Gd}$ & ${ }^{156} \mathrm{Gd}$ & ${ }^{157} \mathrm{Gd}$ & ${ }^{158} \mathrm{Gd}$ \\
\hline Weight [g] & 0.203 & 0.198 & 0.357 & 0.353 \\
Thickness [mm] & 0.1 & 0.1 & 0.2 & 0.2 \\
Isotopic composition [\%] & & & & \\
${ }^{152} \mathrm{Gd}$ & 0.04 & $<0.01$ & $<0.02$ & $<0.1$ \\
${ }^{154} \mathrm{Gd}$ & 0.64 & 0.11 & 0.16 & $<0.1$ \\
${ }^{155} \mathrm{Gd}$ & 91.74 & 1.96 & 0.81 & 0.96 \\
${ }^{156} \mathrm{Gd}$ & 5.11 & 93.79 & 2.21 & 1.7 \\
${ }^{157} \mathrm{Gd}$ & 1.12 & 2.53 & 90.96 & 3.56 \\
${ }^{158} \mathrm{Gd}$ & 0.94 & 1.20 & 5.08 & 92.0 \\
${ }^{160} \mathrm{Gd}$ & 0.41 & 0.41 & 0.80 & 1.82 \\
\hline & & & &
\end{tabular}

hollow Compton-suppression $\mathrm{NaI}(\mathrm{Tl})$ detector with an outer diameter of $33.0 \mathrm{~cm}$ and a length of $35.6 \mathrm{~cm}$. The spectrometer was set in a heavy shield consisting of borated paraffin, borated polyethylene, $\mathrm{Cd},{ }^{6} \mathrm{LiH}$ and potassium free lead [4]. The capture gamma rays were observed at an angle of 125 degree with respect to the proton beam direction. The signals from the spectrometer were recorded in a personal computer as two-dimensional data of pulse-height (PH) and TOF. The measurements with one of the Sn and Gd samples, the ${ }^{197} \mathrm{Au}$ sample, and no sample (Blank) were performed cyclically to average out changes in experimental conditions such as the incident neutron spectrum.

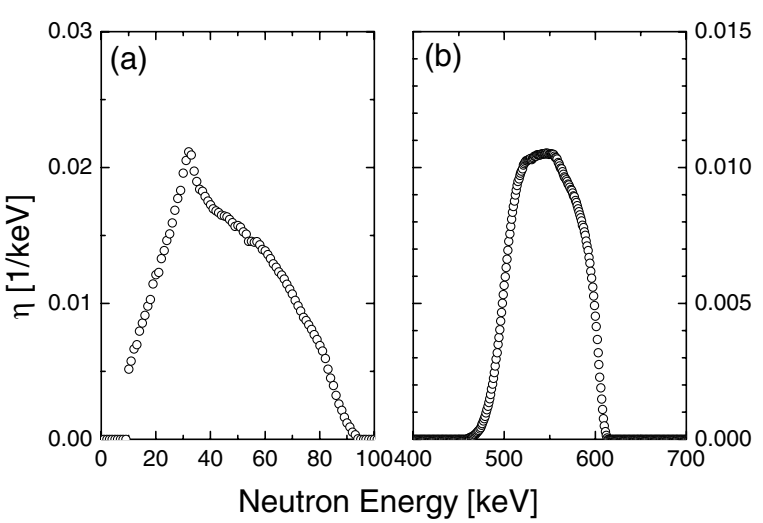

Fig. 2. Typical normalized energy spectra of incident neutrons in the (a) $10-100 \mathrm{keV}$ and (b) $550 \mathrm{keV}$ measurements.

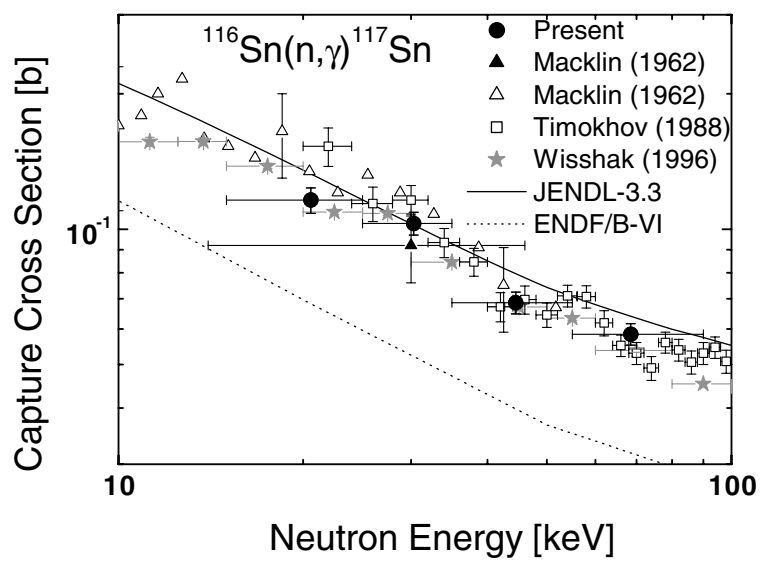

Fig. 3. Neutron capture cross sections of ${ }^{116} \mathrm{Sn}$.

\section{Data processing}

The data processing method has been also given in detail elsewhere [2], so it is summarized here. The incident neutron energy spectrum on the sample was derived from the TOF spectra measured with the ${ }^{6} \mathrm{Li}$-glass detector for the blank run. Typical normalized spectra are shown in figure 2 .

In order to obtain the capture yields of individual samples and the ${ }^{197} \mathrm{Au}$ sample, a PH weighting technique [5] was applied to the net capture gamma-ray $\mathrm{PH}$ spectra. The number of incident neutrons in the ${ }^{197} \mathrm{Au}$ run was determined by the capture yield of ${ }^{197} \mathrm{Au}$ and the averaged capture cross section of ${ }^{197} \mathrm{Au}$, which was obtained from the capture cross sections of ENDF/B-VI [6] and the neutron energy spectrum.

The number of incident neutrons in the $\mathrm{Sn}$ or $\mathrm{Gd}$ isotope run was derived from that in the $\mathrm{Au}$ run and the neutron monitor counts of the ${ }^{6} \mathrm{Li}$-glass detector. The averaged neutron capture cross section of each $\mathrm{Sn}$ or $\mathrm{Gd}$ isotope was derived from the number of incident neutrons and the capture yield of each sample. Corrections for the neutron self-shielding and multiple-scattering in the sample were made by a Monte Carlo method [7], taking account of impurities in the sample. Moreover, other corrections were made for the gamma-ray scattering and absorption in the sample, for the effect of chemical and isotopic impurities in the sample on the capture yields. 


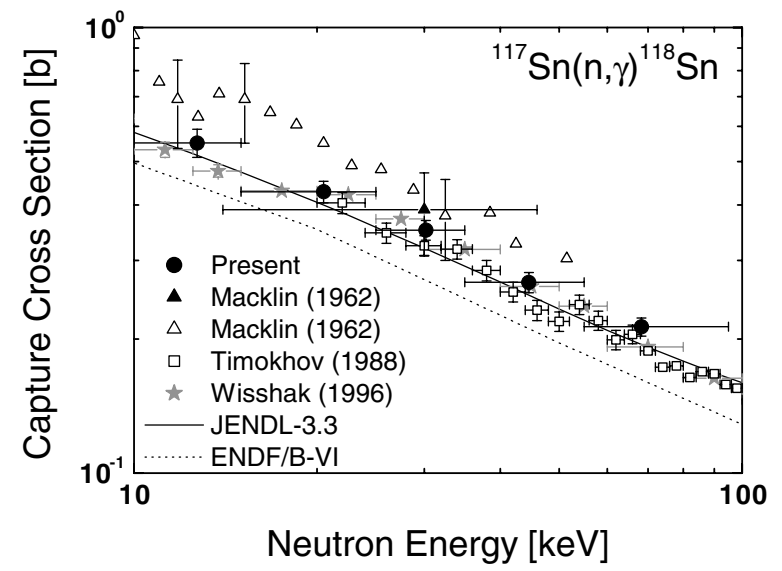

Fig. 4. Neutron capture cross sections of ${ }^{117} \mathrm{Sn}$.

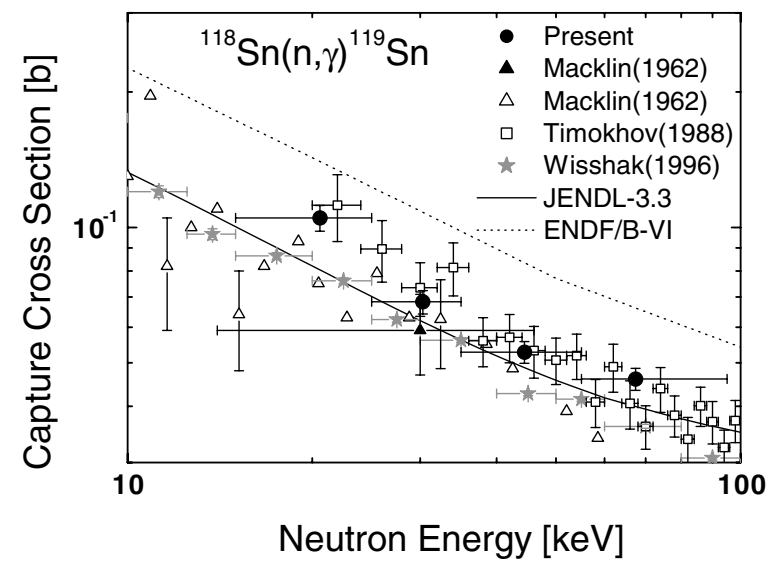

Fig. 5. Neutron capture cross sections of ${ }^{118} \mathrm{Sn}$.

The capture gamma-ray spectra were derived by unfolding the net capture gamma-ray PH spectra with a computer code, FERDOR [8], and the response matrix of the gamma-ray spectrometer.

\section{Results and discussion}

The capture cross sections of ${ }^{117,119} \mathrm{Sn}$ and ${ }^{155,157} \mathrm{Gd}$ were derived with the errors from 4 to $5 \%$ in the energy region from 10 to $100 \mathrm{keV}$ and at $550 \mathrm{keV}$. Those of ${ }^{116,118} \mathrm{Sn}$ and ${ }^{156,158} \mathrm{Gd}$ were derived with the errors from 5 to $8 \%$ in the energy region from 15 to $100 \mathrm{keV}$. The obtained cross sections in the energy region from 10 to $100 \mathrm{keV}$ for $\mathrm{Sn}$ isotopes are shown in figures 3-6, and compared with previous measurements and the evaluations of JENDL-3.3 and ENDF/B-VI.

As shown in figure 3 , the present results of ${ }^{116} \mathrm{Sn}$ are in agreement with those by Macklin et al. [9], Timokhov et al. [10], and Wisshak et al. [11] within the experimental errors. The present results of ${ }^{117,119} \mathrm{Sn}$ in figures 4 and 6 are in agreement with other experimental data within errors. The present results of ${ }^{118} \mathrm{Sn}$ in figure 5 are in good agreement with those by Timokhov et al. [10], but larger than those of Wisshak et al. [11] by about 20\%. The results of Macklin et al. [9] are in agreement with the present ones within their large experimental errors of about $25 \%$. The evaluations of Sn

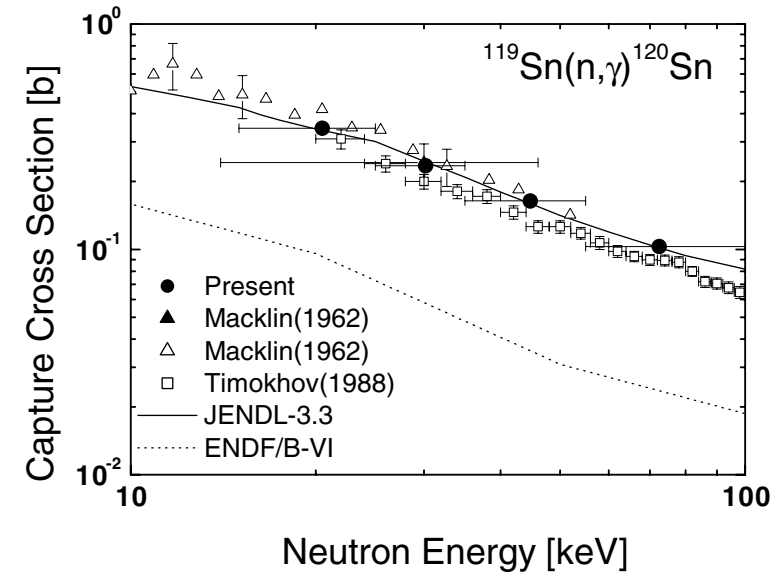

Fig. 6. Neutron capture cross sections of ${ }^{119} \mathrm{Sn}$.

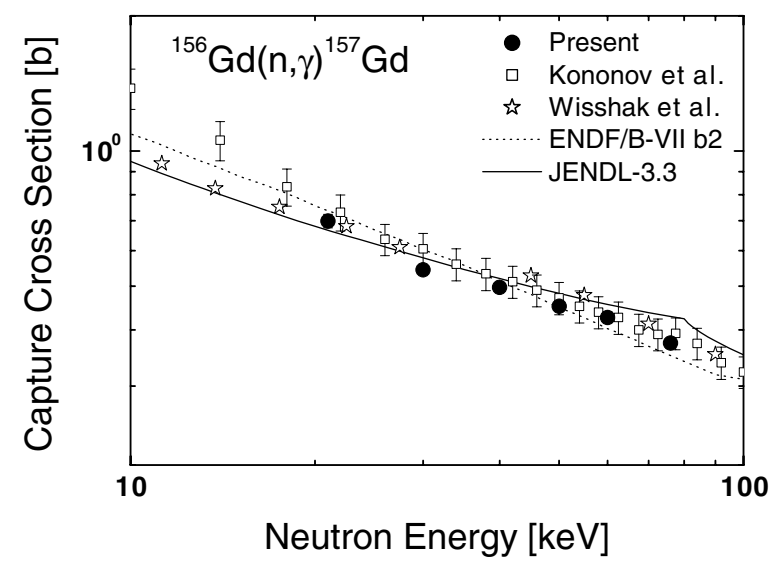

Fig. 7. Neutron capture cross sections of ${ }^{156} \mathrm{Gd}$.

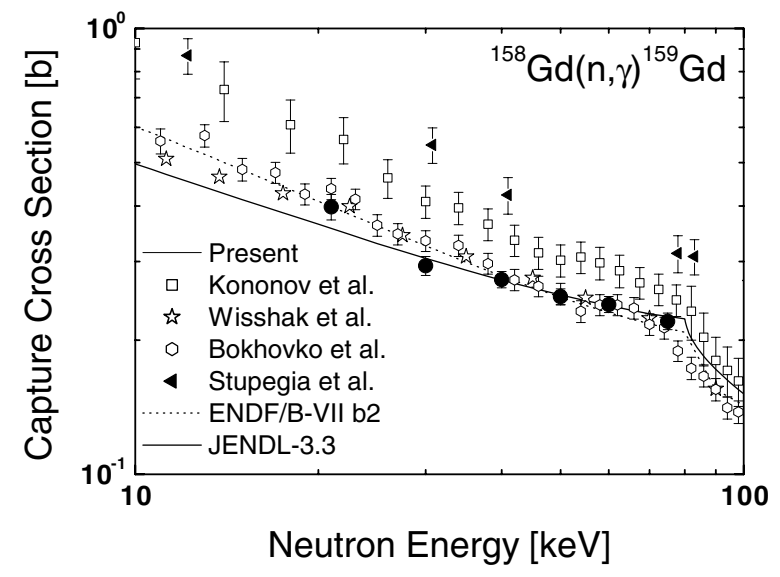

Fig. 8. Neutron capture cross sections of ${ }^{158} \mathrm{Gd}$.

isotopes for ENDF/B-VI [12] were theoretically done before the measurements by Timokhov et al. and Wisshak et al., and the evaluated values of ${ }^{116,117,118,119} \mathrm{Sn}$ are very different from the experiments as shown in figures 3-6. On the other hand, the evaluated values of JENDL-3.3 for Sn isotopes [13] are in agreement with the experimental within $20 \%$, because 


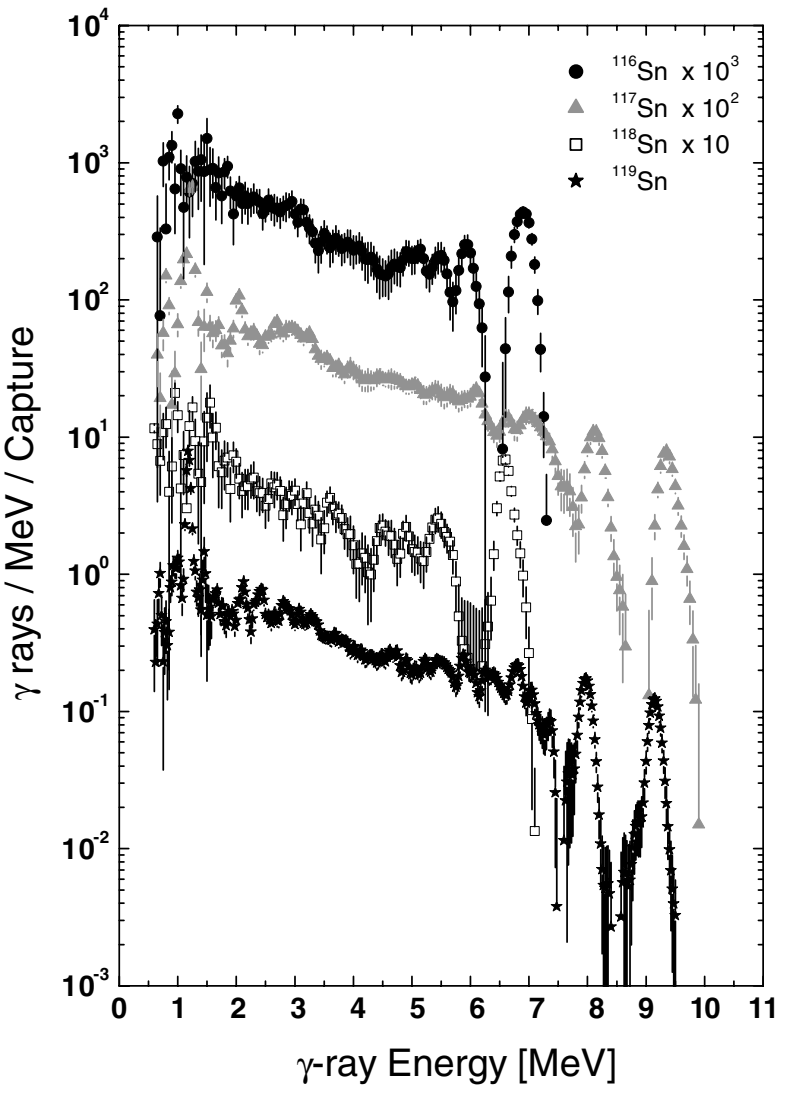

Fig. 9. The neutron capture gamma-ray spectra of ${ }^{116,117,118,119} \mathrm{Sn}$ in the neutron energy region from 15 to $100 \mathrm{keV}$.

the evaluated values were normalized to reproduce experimental data at several tens of keV. For example, the evaluated values of JENDL-3.3 for ${ }^{116} \mathrm{Sn}$ were adjusted to reproduce the capture cross section of $85 \mathrm{mb}$ at $40 \mathrm{keV}$ measured by Macklin, and are larger than the present ones by 10-15\%, except for the values around $30 \mathrm{keV}$. It means that improvement of theoretical calculation of neutron capture reaction is important for the accurate evaluation of neutron capture cross sections of LLFPs for which measurements are difficult.

The obtained cross sections in the energy region from 10 to $100 \mathrm{keV}$ for ${ }^{156,158} \mathrm{Gd}$ are shown in figures 7 and 8 , and compared with previous measurements and the evaluations of JENDL-3.3 and ENDF/B-VII-b2. The results of ${ }^{155,157} \mathrm{Gd}$ have been published elsewhere [14]. As shown in figure 7, the present results for ${ }^{156} \mathrm{Gd}$ are in general agreement with those of Kononov et al. [15] and Wisshak et al. [16] and with the evaluated values in ENDF/B-Z-b2 [17] and JENDL-3.3 [18]. The present results for ${ }^{158} \mathrm{Gd}$ in figure 8 agree with those of Wisshak et al. [16] and Bokhovko et al. [19] and with the evaluated values in both ENDF/B-VII-b2 [17] and JENDL 3.3 [18]. However, the experimental values of Kononov et al. [15] and Stupegia et al. [20] are larger than any other values.

The capture gamma-ray spectra obtained from the measurements for the ${ }^{116,117,118,119} \mathrm{Sn}$ samples are shown in figure 9, each spectrum has strong transitions from the neutron capture state to the ground state, the first excited state and other low-lying states.

\section{Conclusion}

The neutron capture cross sections and capture gamma-ray spectra of ${ }^{116-119} \mathrm{Sn}$ and ${ }^{155-158} \mathrm{Gd}$ have been measured in the incident neutron energy region from 10 to $100 \mathrm{keV}$ and at $550 \mathrm{keV}$. These data will provide important information on nuclear reaction mechanisms and nuclear excitation modes, and the information will be used for accurate evaluations of the neutron capture cross sections of important nuclide such as ${ }^{126} \mathrm{Sn}$.

The present study on Sn isotopes is supported by a Grant-in-Aid of the Japan Ministry of Education, Culture, Sports, Science and Technology. And the research on Gd isotopes is partly supported through the Long-term Nuclear R\&D program of the Korea Atomic Energy Research Institute (KAERI). One of the authors (G. Kim) is partly supported by the Science Research Center (SRC) program of the Institute of High Energy Physics, Kyungpook National University.

\section{References}

1. S. Zhang et al., Radiochim. Acta 94, 385 (2006).

2. S. Mizuno et al., J. Nucl. Sci. Technol. 36, 493 (1999).

3. K. Ohgama, Doctoral thesis, Tokyo Institute of Technology, (2005).

4. M. Igashira, H. Kitazawa, N. Yamamuro, Nucl. Instrum. Meth. A 245, 432 (1986).

5. R.L. Macklin, J.H. Gibbons, Phys. Rev. 159, 1007 (1967).

6. ENDF/B-VI data file for ${ }^{197} \mathrm{Au}(\mathrm{MAT}=7925)$, evaluated by P.G. Young (1991).

7. K. Senoo et al., Nucl. Instrum. Meth. A 339, 556 (1994).

8. H. Kendrick, S.M. Sperling, An Introduction to the Principles and Use of the FERDOR Code, Gulf Radiation Technology, GA9882 (1970).

9. R.L. Macklin, T. Inada, J.H. Gibbons, Nature 194, 1272 (1962).

10. V.M. Timokhov et al., Sov. J. Nucl. Phys. 50, 375 (1989).

11. K. Wisshak et al., Phys. Rev. C 54, 1451 (1996).

12. ENDF/B-VI data file for ${ }^{116} \mathrm{Sn}(\mathrm{MAT}=5037),{ }^{117} \mathrm{Sn}(\mathrm{MAT}=$ $5040),{ }^{118} \mathrm{Sn}(\mathrm{MAT}=5043)$, and ${ }^{119} \mathrm{Sn}(\mathrm{MAT}=5046)$, evaluated by R.E. Schenter, F. Schmittroth (1974).

13. JENDL-3.3 data file for ${ }^{116} \mathrm{Sn}(\mathrm{MAT}=5037),{ }^{117} \mathrm{Sn}(\mathrm{MAT}=$ $5040),{ }^{118} \mathrm{Sn}(\mathrm{MAT}=5043)$, and ${ }^{119} \mathrm{Sn}(\mathrm{MAT}=5046)$, evaluated by JNDC FP Nuclear Data W.G. (1990).

14. W.C. Chung et al., J. Kor. Phys. Soc. 50, 409 (2007).

15. V.N. Kononov et al., Sov. J. Nucl. Phys. 27, 5 (1978).

16. K. Wisshak et al., Phys. Rev. C 52, 2762 (1995).

17. D. Rochman et al., http://www.nndc.bnl.gov/exfor2/endf00.htm, ENDF/B-VIIb2-300 data file for ${ }^{156} \mathrm{Gd}(\mathrm{MAT}=6437)$ and ${ }^{158} \mathrm{Gd}$ $(\mathrm{MAT}=6443)(2006)$.

18. JENDL-3.3 data file for ${ }^{156} \mathrm{Gd}(\mathrm{MAT}=6437)$ and ${ }^{158} \mathrm{Gd}(\mathrm{MAT}=$ 6443), evaluated by JNDC FP Nuclear Data W.G. (2002).

19. M.V. Bokhovko et al., Yadernye Konstanty 2, 44 (1985).

20. D.C. Stupegia et al., J. Nucl. Energy 22, 267 (1968). 